\title{
Notification that New Names and New Combinations Have Appeared in Volume 47, No. 1, of the $\operatorname{IJSB}^{a}$
}

\begin{tabular}{|c|c|c|c|}
\hline Name: & Proposed as: & Authors: & $\begin{array}{l}\text { IJSB reference } \\
\text { description: }\end{array}$ \\
\hline Vogesella & gen. nov. & Grimes et al. & 47(1):25 \\
\hline Vogesella indigofera (basonym Pseudomonas indigofera) & comb. nov. & Grimes et al. & 47(1):25 \\
\hline Arcanobacterium bernardiae (basonym Actinomyces bernardiae) & comb. nov. & Pascual Ramos et al. & 47(1):51 \\
\hline Arcanobacterium pyogenes (basonym Actinomyces pyogenes) & comb. nov. & Pascual Ramos et al. & 47(1):51 \\
\hline Arcanobacterium phocae & sp. nov. & Pascual Ramos et al. & 47(1):52 \\
\hline Vibrio scophthalmi & sp. nov. & Cerdà-Cuéllar et al. & $47(1): 60$ \\
\hline Treponema medium & sp. nov. & Umemoto et al. & 47(1):71 \\
\hline Haloarcula argentinensis & sp. nov. & Ihara et al. & 47(1):76 \\
\hline Haloarcula mukohataei & sp. nov. & Ihara et al. & 47(1):76 \\
\hline Spiroplasma diabroticae & sp. nov. & Carle et al. & $47(1): 80$ \\
\hline Mycobacterium hassiacum & sp. nov. & Schröder et al. & 47(1):90 \\
\hline Conynebacterium coyleae & sp. nov. & Funke et al. & 47(1):94 \\
\hline Spirilliplanes & gen. nov. & Tamura et al. & 47(1):101 \\
\hline Spirilliplanes yamanashiensis & sp. nov. & Tamura et al. & 47(1):102 \\
\hline Moraxella boevrei & sp. nov. & Kodjo et al. & 47(1):120 \\
\hline Thermonema rossianum & sp. nov. & Nobre et al. ${ }^{b}$ & $47(1): 125$ \\
\hline Skermania & gen. nov. & Chun et al. & 47(1):129 \\
\hline Skermania piniformis (basonym Nocardia pinensis) & comb. nov. & Chun et al. & 47(1):129 \\
\hline Desulfonatronovibrio & gen. nov. & Zhilina et al. & $47(1): 149$ \\
\hline Desulfonatronovibrio hydrogenovorans & sp. nov. & Zhilina et al. & $47(1): 149$ \\
\hline Anaerobaculum & gen. nov. & Rees et al. & 47(1):153 \\
\hline Anaerobaculum thermoterrenum & sp. nov. & Rees et al. & 47(1):153 \\
\hline Schwartzia & gen. nov. & van Gylswyk et al. & 47(1):158 \\
\hline Schwartzia succinivorans & sp. nov. & van Gylswyk et al. & 47(1):158 \\
\hline Stenotrophomonas africana & sp. nov. & Drancourt et al. & $47(1): 162$ \\
\hline Clostridium pascui & sp. nov. & Wilde et al. & 47(1):169 \\
\hline Sphingomonas aromaticivorans & sp. nov. & Balkwill et al. & 47(1):199 \\
\hline Sphingomonas subterranea & sp. nov. & Balkwill et al. & 47(1):199 \\
\hline Sphingomonas stygia & sp. nov. & Balkwill et al. & 47(1):199 \\
\hline Blastochloris & gen. nov. & Hiraishi & 47(1):218 \\
\hline Blastochloris viridis (basonym Rhodopseudomonas viridis) & comb. nov. & Hiraishi & $47(1): 218$ \\
\hline Blastochloris sulfoviridis (basonym Rhodopseudomonas sulfoviridis) & comb. nov. & Hiraishi & 47(1):218 \\
\hline Flammeovirga & gen. nov. & Nakagawa et al. & 47(1):221 \\
\hline Flammeovirga aprica (basonym Cytophaga aprica) & comb. nov. & Nakagawa et al. & 47(1):221 \\
\hline Persicobacter & gen. nov. & Nakagawa et al. & 47(1):221 \\
\hline Persicobacter diffluens (basonym Cytophaga diffluens) & comb. nov. & Nakagawa et al. & $47(1): 222$ \\
\hline
\end{tabular}

${ }^{a}$ This listing of names published in a previous issue of IJSB is provided as a service to bacteriology to assist in the recognition of new names and new descriptions. This procedure was proposed by the Judicial Commission [Minute II (ii), Int. J. Syst. Bacteriol. 41:185, 1991]. The names given herein have priority according to the issue of the IJSB in which they were published.

${ }^{b}$ Complete authorship reads: Nobre, Rainey, and da Costa in Tenreiro, Nobre, Rainey, Miguel, and da Costa. 